\title{
Model, Metamodel and Topology
}

\author{
J. Nescolarde-Selva \\ Department of Applied Mathematics. University of Alicante. Alicante. Spain. \\ J.L. Usó-Doménech \\ Department of Mathematics. University Jaume I. Castelló de la Plana. Spain.
}

\begin{abstract}
This reply to Gash's (2013) commentary on Nescolarde-Selva and UsóDomènech (Semiotic Vision of Ideologies) answers the three questions raised and at the same time opens up new questions.
\end{abstract}

Keywords Belief materialization, Dynamics of Systems, Probabilistic model, Substantive beliefs, Values

In his commentary on our paper, Professor Gash (2013) raises three interesting questions. In fact, it was not our intention in writing the paper to present a particular model, but a metamodel, that is, the epistemological basis of a model covering aspects of human society., In so far as we know, mathematics has not yet entered too strongly into belief systems in general, and in particular into ideologies. These epistemological considerations form the basis, the foundation of an abstract formal model, which we have designated (non perjoratively) as a Dogmatic System (Usó-Domènech and Nescolarde-Selva, 2012 ${ }^{\mathrm{a}}$ ). This formal model, that involves time, i.e., is diachronic, but still is formal. Its variables are abstract, indicating no "real" variables. Its foundation is an adaptation to human society of Patten's Environ Theory for ecosystems (Patten, 1978). Following this prior brief introduction, we briefly answer the three interesting issues raised by Professor Gash, although for obvious reasons we changed the order of them.

1) Is it possible to show which conditions might change so that this individual's thinking could change? Related to this question is the additional one as to how the model functions across time (diachronically) as opposed to at one moment of time (synchronically)? A formal model can be converted into an operational model. System Dynamics (Forrester, 1961) suggest a system of equations of state (differential equations function of time). Differential equations correspond to the state variables. The ordinary differential equations of state variables contain the input and output flow equations by unit of time of each level of compartment. These models have been used to study the behavior of ecosystems (Usó-Doménech et al., 1995; Usó-Doménech et al., 1997) but nothing prevents use for human ecosystem. The variables will be different, with different field work, but the methodology will be the same. This type of operative model allows simulations with different scenarios, on the upper and lower limits the best and the worst of scenarios respectively. But it has a limitation. Modeling individuals is impossible and requires populations, and its accuracy will be larger the larger the population. Individual behaviour cannot be predicted with any existing and possible future model.

2) Will the mathematical models proposed function more effectively with probabilistic approaches to interpretation? Yes. Operating models as explained in the previous paragraph can be adapted to probabilistic functions. The state 
equations can be stochastic differential equations and the flow equations (inputs) carry an associated probability. The flow equations (outputs) lead also an associated probability. The only drawback is simply operating. It requires fieldwork with successive surveys to calculate the probability of each trend. This requires time, dedication and money.

3) Does this systemic model of belief systems help to understand the conditions leading another person to "engage in discussion to understand differences between their own and another's view"? We think it was Hesiod who said "The sun shines equally in Persia and Hellas, but the concept of good and evil are different." LeShan and Margenau (1982) argue the existence of a parallel between the structures of science and ethics: laws are similar and compatible, but the observables are different. In the scientific domain observables that we consider are those relating to the physical and biological sciences: position, temperature, valence, genetic structure etc. In ethics, the observables are the values that are designated by a variety of names. Verification in science involves postulate certain rules. Science begins and ends with human choices. The structure of ethics is formally similar, but the language is, of course, different. While science, describing facts is somehow tied to the indicative mood of language, ethics, dedicated to prescribe and prohibit human actions must use imperatives. The action taken by a certain group of people, a tribe, nation or culture, resulting in specific forms of culture, generating constructions commonly called "values." These values, which automatically are born of obedience to the imperatives are the "values of fact." The truth is that the values in fact lack normative force, as being merely in fact lack the "should." We must consider under what conditions "to be" deserves and acquires the authority of a "should be", how to reach ethics "validity", which is the counterpart of "reality" in science. The achievement of the validity involves establishing isomorphisms with another set of values called ideal values. Any system of ethics contains, besides their corresponding rules and standards of conduct, a set of goals that are the ideal values. These values are almost universally accepted in almost all cultures containing maxims on personal and collective human happiness, freedom of action or belief, life, health, and sometimes intimacy. It almost universality suggests divine inspiration as in the case of the striking similarity of the imperatives. That is why we are placed in what we call a Mythical Superstructure. These ideal values are derived from substantive beliefs characteristic of a particular culture. Ideal values and myth (as residual ideology) belong to Mythical Superstructure (MS). Its projection conforms, with the image coming from the Structural Base (SB), the dominant ideology and the values in fact of the Ideological Doxical Superstructure (IDS), and this projects on the SB in forms of actions and conducts that are reflected on the Mythical Superstructure (MS) like utopia, the last objective of the ideology (UsóDomènech and Nescolarde-Selva, 2012 ${ }^{\mathrm{b}}$ ). We have tried to demonstrate (UsóDomènech and Nescolarde-Selva, 2012 ${ }^{\mathrm{c}}$ ) that these connected beliefs have a mathematical structure, a topological structure concretely. The ideas and beliefs are expressed inarchitecture and in written, pictorial, musical, etc., texts. Literary speech, architecture, artistic styles, are differentiated clearly according to historical times, corresponding to the world visions of the people who lived in those periods. These cultural products are, in fact, materializations of abstract belief systems and nobody can deny that all of them have a geometric, topological structure. And in using mathematical structures, we have reached 
the following conclusions: Let $\mathrm{L}$ be a language. We suppose the existence of $\mathrm{n}$ substantive beliefs $s_{1}, s_{2}, \ldots, s_{n}$ coexisting at a certain historical moment. Let $ב$ be the set of all substantive beliefs such that $\Sigma=\left\{s_{1}, s_{2}, \ldots, s_{n}\right\}$. Let $\varepsilon$ be a sentence such that $\varepsilon \in s_{i}, s_{i} \in \Sigma$. A set of substantive beliefs $\mathrm{S} \subset \Sigma$ is called open, if for each $\mathrm{s} \in \mathrm{S}$ there exists and $\varepsilon \neq \varnothing$ such that the interval ( $\mathrm{s}-\varepsilon, \mathrm{s}+$ $\varepsilon$ ) is contained in $S$. A set $S$ of substantive beliefs is called closed if the complement of $\mathrm{S}, \mathrm{BS} \backslash \mathrm{S}$, is open. Closed sets $S$ correspond to belief systems ideologically closed and impermeable, such as dogmatic religions or political totalitarian ideologies.

If topological belief structures are embodied in art, text, etc. then why cannot we think of form of topological structure in the brain of the believer? At the same time, the topological cerebral structure, won't this be reflected in the belief system? Here's a cybernetic system with successive feedbacks. The issue is open and only by approaching it can one avoid one of the major problems that humanity has been and is: fanaticism and intolerance. Both lead to the Holocaust.

\section{References}

Forrester, J.W. Industrial Dynamics. MIT Press. Cambridge, MA.

Gash, H. 2013. Fixed or Probable Ideas? Foundations of Science. DOI 10.1007/s10699013-9330-2.

LeShan, L. and Margenau, H. 1982. Einstein's Space and Van Gogh's Sky. MacMillan Publ. Co. Inc. New York.

Patten, B.C. 1978. Systems approach to the concept of environment. Ohio Journal of Science. 78(4), 206-222.

Usó-Doménech, J.L., Villacampa-Esteve, Y., Stübing-Martinez, G., Karjalainen, T. and Ramo, M.P. 1995. MARIOLA: a model for calculating the response of mediterranean bush ecosystem to climatic variations. Ecological Modelling, 80, 113-129.

Nescolarde-Selva, J. A., and Usó-Doménech, J. 2013. Semiotic vision of ideologies. Foundations of Science. DOI:10.1007/s10699-013-9329-8.

Usó-Doménech, J.L., Mateu, J. and López, J.A. 1997. Mathematical and statical formulation of an ecological model with applications. Ecological Modelling, 101, 2740 .

Usó-Doménech, J.L. and Nescolarde-Selva, J. 2012 ${ }^{\mathrm{a}}$. Mathematic and Semiotic Theory of Ideological System. A Systemic vision of Beliefs. Cap 13. Lambert Academic Publishing. Saarbrücken. Germany. 
Usó-Doménech, J.L. and Nescolarde-Selva, J. 2012 ${ }^{\mathrm{b}}$. Mathematic and Semiotic Theory of Ideological System. A Systemic vision of Beliefs. Cap 6. Lambert Academic Publishing. Saarbrücken. Germany.

Usó-Doménech, J.L. and Nescolarde-Selva, J. 2012 ${ }^{\mathrm{c}}$. Mathematic and Semiotic Theory of Ideological System. A Systemic vision of Beliefs. Caps 7, 11 and 12. Lambert Academic Publishing. Saarbrücken. Germany. 